\title{
A Smartphone Interface for a Wireless EEG Headset with Real-Time 3D Reconstruction
}

\author{
Stopczynski, Arkadiusz; Larsen, Jakob Eg; Stahlhut, Carsten; Petersen, Michael Kai; Hansen, Lars Kai
}

Published in:

Affective Computing and Intelligent Interaction

Link to article, DOI:

10.1007/978-3-642-24571-8_40

Publication date:

2011

Link back to DTU Orbit

Citation (APA):

Stopczynski, A., Larsen, J. E., Stahlhut, C., Petersen, M. K., \& Hansen, L. K. (2011). A Smartphone Interface for a Wireless EEG Headset with Real-Time 3D Reconstruction. In S. D Mello (Ed.), Affective Computing and Intelligent Interaction: 4th International Conference, ACll 2011 Memphis, TN, USA, October 9-12, 2011 Proceedings, Part II (pp. 317-318). Springer. Lecture Notes in Computer Science No. 6975 https://doi.org/10.1007/978-3-642-24571-8_40

\section{General rights}

Copyright and moral rights for the publications made accessible in the public portal are retained by the authors and/or other copyright owners and it is a condition of accessing publications that users recognise and abide by the legal requirements associated with these rights.

- Users may download and print one copy of any publication from the public portal for the purpose of private study or research.

- You may not further distribute the material or use it for any profit-making activity or commercial gain

- You may freely distribute the URL identifying the publication in the public portal 


\title{
A smartphone interface for a wireless EEG headset with real-time $3 \mathrm{D}$ reconstruction
}

\author{
Arkadiusz Stopczynski, Jakob Eg Larsen, Carsten Stahlhut, \\ Michael Kai Petersen, and Lars Kai Hansen \\ DTU Informatics, Cognitive Systems \\ Technical University of Denmark, Building 321, DK-2800 Kgs. Lyngby, Denmark \\ \{arks, jel, cs , mkp, lkh\}@imm.dtu.dk
}

\begin{abstract}
We demonstrate a fully functional handheld brain scanner consisting of a low-cost 14-channel EEG headset with a wireless connection to a smartphone, enabling minimally invasive EEG monitoring in naturalistic settings. The smartphone provides a touch-based interface with real-time brain state decoding and $3 \mathrm{D}$ reconstruction.
\end{abstract}

\section{Introduction}

Functional brain imaging techniques including fMRI and EEG provide moving picture access to the living human brain, however, relying on complex heavy hardware they offer very limited comfort for the user, and thus can not be used under naturalistic conditions. This induce largely unknown biases into the current state of the art brain scanning, thought to be particularly problematic for studies of emotion and social cognition. Cap-based EEG systems allow more naturalistic conditions but typically limiting movement due to wiring. There are obvious advantages of brain monitoring under naturalistic conditions [1], in order to understand how we perceive our surroundings in mobile real-life settings. We demonstrate a minimally invasive and mobile brain monitoring system (see Fig. 1) that enables real-time brain state decoding and 3D cortical activity visualization within a low-cost highly mobile smartphone environment.
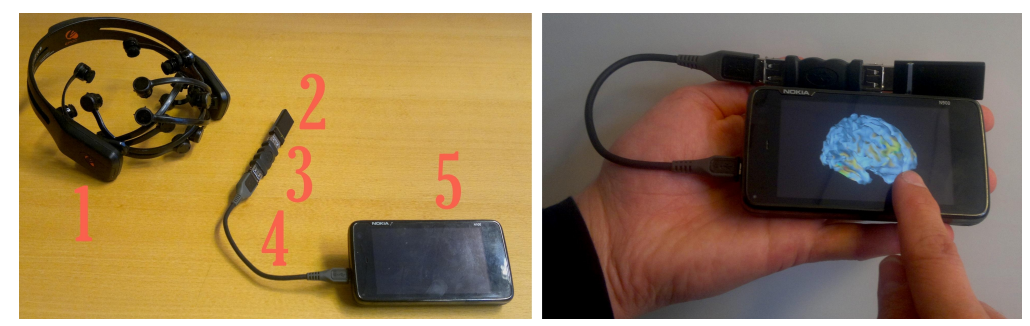

Fig. 1. (left) The system with Emotiv EPOC wireless EEG headset (1), Receiver module with USB connector (2), USB connector and adapter $(3+4)$, and Nokia N900 (5). (right) Touch-based interaction with a $3 \mathrm{D}$ model of the brain using the smartphone. 
EEG biofeedback has shown to produce behavioral effects including improvements in reaction times, emotional responses, and musical performance. Clinical applications include attention-deficit and epilepsy, for a review see [2]. Such applications will benefit from a low-cost and easy-to-use personal brain monitor.

\section{System Architecture}

Our system provides a fully portable EEG based real-time functional brain scanner including stimulus delivery, data acquisition, logging, brain state decoding and 3D activity visualization. The raw EEG data is obtained from a wireless Emotiv 14 channel Neuroheadset with a sampling rate of $128 \mathrm{~Hz}$ and electrodes positioned at AF3, F7, F3, FC5, T7, P7, O1, O2, P8, T8, FC6, F4, F8, AF4 (the international 10-20 system). The headset transmits the EEG data to a receiver module connected to a Nokia N900 phone. Custom-made software is used on the phone to do real-time transmission of the EEG data to a server and for local processing, enabling real-time brain state decoding and a rich feedback signal to the user through a 3D rendering of the active cortical EEG sources. The user can interact with the 3D model of the brain by touch gestures (see Fig. 1).

\section{Evaluation}

A major concern in mobile real-time systems is the power consumption, hence, battery life. Our experiments with local logging of EEG data allowed 7.5 hours continous usage, whereas remote logging allowed 3.5 hours. The $3 \mathrm{D}$ brain model contains 5124 vertices and 10240 triangles and is stored in the mobile application and brain activity is reflected by changing colors, allowing rendering performance of approximately $30 \mathrm{fps}$ and fluent touch-based interaction with the 3D model. The current design of the system has a delay of approximately $150 \mathrm{~ms}$. between the signal appearing in the brain and being visualized on the smart phone.

Our evaluations have demonstrated the potential for minimally invasive and low-cost EEG monitoring in naturalistic settings, as the functional brain scanner allow the user to do both long-term recordings and real-time monitoring of own $3 \mathrm{D}$ brain activity patterns on a smartphone.

Supporting Material. http://milab.imm.dtu.dk/eeg

Acknowledgments. This work is supported in part by the Danish Lundbeck Foundation through CIMBI Center for Integrated Molecular Brain Imaging.

\section{References}

1. S. Makeig, K. Gramann, T.P. Jung, T.J. Sejnowski, and H. Poizner, "Linking brain, mind and behavior" Int J Psychophysiol, vol. 73, no. 2, pp. 95100, 2009.

2. N. Weiskopf, F. Scharnowski, R. Veit, R. Goebel, N. Birbaumer, and K. Mathiak, "Self-regulation of local brain activity using real-time functional magnetic resonance imaging (fmri)" J Physiol Paris, 2005. 


\section{Description of the technical set-up and requirements}

Our demonstration will allow participants to try the handheld brain scanner setup including the wireless EEG headset and the smartphone.

Specific requirements for the demo setup include:

- Table and two chairs

- Power supply with at least 6 outlets

- Wireless network access - preferable the possibility to setup our own wireless router enabling the communication (to a server) needed in our system. 